\title{
The Human Ventromedial Prefrontal Cortex: Sulcal Morphology and Its Influence on Functional Organization
}

\author{
[DAlizée Lopez-Persem, ${ }^{1}$ ㄴennart Verhagen, ${ }^{1}$ @Céline Amiez, ${ }^{2}$ Michael Petrides, ${ }^{3}$ and ${ }^{-}$Jérôme Sallet ${ }^{1}$ \\ ${ }^{1}$ Wellcome Integrative Neuroimaging Centre, Department of Experimental Psychology, University of Oxford, Oxford OX1 3SR, United Kingdom, ${ }^{2}$ University \\ of Lyon, Université Lyon 1, INSERM, Stem Cell and Brain Research Institute U1208, 69500 Bron, France, and 3Montreal Neurological Institute, McGill \\ University, Montreal, Quebec H3A2B4, Canada
}

The ventromedial prefrontal cortex (vmPFC), which comprises several distinct cytoarchitectonic areas, is a key brain region supporting decision-making processes, and it has been shown to be one of the main hubs of the Default Mode Network, a network classically activated during resting state. We here examined the interindividual variability in the vmPFC sulcal morphology in 57 humans (37 females) and demonstrated that the presence/absence of the inferior rostral sulcus and the subgenual intralimbic sulcus influences significantly the sulcal organization of this region. Furthermore, the sulcal organization influences the location of the vmPFC peak of the Default Mode Network, demonstrating that the location of functional activity can be affected by local sulcal patterns. These results are critical for the investigation of the function of the vmPFC and show that taking into account the sulcal variability might be essential to guide the interpretation of neuroimaging studies.

Key words: Default Mode Network; function; morphology; sulcus; vmPFC

\section{Significance Statement}

The ventromedial prefrontal cortex (vmPFC) is one of the main hubs of the Default Mode Network and plays a central role in value coding and decision-making. The present study provides a complete description of the interindividual variability of anatomical morphology of this large portion of prefrontal cortex and its relation to functional organization. We have shown that two supplementary medial sulci predominantly determine the organization of the vmPFC, which in turn affects the location of the functional peak of activity in this region. Those results show that taking into account the variability in sulcal patterns might be essential to guide the interpretation of neuroimaging studies of the human brain and of the vmPFC in particular.

\section{Introduction}

The ventromedial prefrontal cortex (vmPFC) is a key region for decision-making and subjective valuation processes (Lebreton et

Received Aug. 11, 2018; revised Nov. 21, 2018; accepted Jan. 9, 2019.

Author contributions: A.L.-P., C.A., M.P., and J.S. designed research; A.L.-P. and J.S. performed research; A.L.-P. and L.V. contributed unpublished reagents/analytic tools; A.L.-P. analyzed data; A.L.-P., L.V., M.P., and J.S. wrote the paper.

Data were provided by the Washington University-University of Minnesota Consortium of the Human Connectome Project (Principal Investigators: David Van Essen and Kamil Ugurbil; Grant 1U54MH091657) funded by the 16 institutes of the National Institutes of Health (NIH) and Centers that support the NIH Blueprint for Neuroscience Research; and by the McDonnell Center for Systems Neuroscience at Washington University. A.L.-P. received a Fellowship from the Fondation pour la Recherche Medicale. J.S. was supported by a Sir Henry Dale Wellcome Trust Fellowship (105651/Z/14/Z). The Wellcome Centre for Integrative Neuroimaging is supported by core funding from the Wellcome Trust (Grant 203139/Z/16/Z).

The authors declare no competing financial interests.

Correspondence should be addressed to Alizée Lopez-Persem at lopez.alizee@gmail.com or Jérôme Sallet at Jerome.sallet@psy.ox.ac.uk.

https://doi.org/10.1523/JNEUROSCI.2060-18.2019 Copyright $@ 2019$ Lopez-Persem et al.

This is an open-access article distributed under the terms of the Creative Commons Attribution License Creative Commons Attribution 4.0 International, which permits unrestricted use, distribution and reproduction in any medium provided that the original work is properly attributed. al., 2009; Noonan et al., 2010; Lopez-Persem et al., 2016). This brain region is not only involved in reward and value processing but also in emotional regulation (Hänsel and von Känel, 2008) and memory representation (Bonnici et al., 2012), and is a main hub of the Default Mode Network (DMN; Raichle, 2015), a network classically observed during resting state (rs) and mind wandering. Thus, vmPFC function is now the focus of many studies.

Defining the functional contributions of a cortical territory first requires an understanding of its anatomical organization. The cytoarchitectonic areas found in a region have been shown to have distinct connectional profiles with other cortical and subcortical areas and also to relate to separate functional processes (Petrides, 1996, 2002). Indeed, the functional contributions of brain areas are constrained by their inputs and outputs (i.e., their connectivity patterns; Passingham et al., 2002; Petrides, 2002). These cytoarchitectonic areas often relate to sulcal patterns that can be clearly visualized in functional imaging studies.

Characterizing the functions of the vmPFC is challenging for two reasons. First, the term "vmPFC" does not refer to a specific anatomically delineated brain area. In functional neuroimaging 
studies that are based on the average brain activity of a group, the term vmPFC has been used to label a large portion of the prefrontal cortex (Bartra et al., 2013) that includes several cytoarchitectonic areas (i.e., areas 10, 14, 25, and 32; Fig. 1), and vmPFC boundaries are debated (Wallis, 2011). The second challenge in assigning a function to the different subdivisions of the vmPFC comes from the fact that the classic neuroimaging approach is to average results across subjects in the Montreal Neurological Institute (MNI) stereotactic space (Evans et al., 1993). This approach ignores the considerable interindividual variability in the morphological sulcal patterns of the vmPFC (Chiavaras and Petrides, 2000; Mackey and Petrides, 2014). It should be emphasized here that there is evidence in the vmPFC that cytoarchitectonic areas relate well to particular sulci (Mackey and Petrides, 2014).

Sulcal patterns could indeed vary either in terms of the presence/absence of certain sulci or in terms of the shape/relative position of sulci. Importantly, it has been shown that the locations of distinct experienced value signals could be predicted from the organization of the sulci in the orbitofrontal cortex (Li et al., 2015). Moreover, taking into account the variability in sulcal patterns has proven to be essential to the interpretation of neuroimaging studies in the lateral prefrontal cortex and the cingulate cortex (Amiez et al., 2006, 2013). Thus, differences in functional properties in the vmPFC could also be related to morphological heterogeneity across individuals.

In the present study, we first provide a precise description of the sulci of the vmPFC, and we then show that distinct morphological patterns exist between individuals, preventing exact morphological alignment across individuals, and how those patterns affect the sulcal organization of the vmPFC. Finally, we show that this information is relevant to the study of the function of the vmPFC through a link between sulcal anatomy and the local response of the Default Mode Network.

\section{Materials and Methods}

Subjects. The data used in this study are released as part of the Human Connectome Project (HCP; Washington University-University of Minnesota Consortium of the Human Connectome Project; RRID: SCR_008749; http://db.humanconnectome.org; Van Essen et al., 2012). We selected the $S 900$ subjects release with $7 \mathrm{~T}$ structural and resting-state fMRI data. The data were preprocessed according to the HCP pipeline (Glasser et al., 2013). All analyses were conducted on the data aligned using areal feature-based registration (called "MSMAll" for "Multimodal Surface Matching"; Glasser et al., 2016). This procedure aligns vertices on the cortical surface across subjects not only according to gross folding morphology, but also taking into account the subject-specific functional features, such as the location and distribution of resting-state networks. The MSMAll approach dramatically improves the functional alignment of cortical areas over and above registration based solely on volumetric or surface-based morphological registration. This type of registration is referred to as "area-based" registration and is sometimes considered a near optimal functional alignment (Glasser et al., 2016). Here we test whether considering detailed interindividual variability in sulcal patterns can ex- plain functional organization even beyond this state-of-the-art areabased alignment approach. Of the 73 subjects in this specific HCP release, 16 subjects were excluded because of family ties with other subjects in the database. The data analysis was therefore based on 114 hemispheres of 57 subjects ( 37 females).

Sulcal identification. The analyses were restricted to the ventral part of the medial wall of the prefrontal cortex, delineated by an arbitrary horizontal line that runs from the front of the brain to the genu of the corpus callosum. This delineation allowed us to define a broad anatomical mask used in the resting-state analysis. Following Mackey and Petrides (2014), we identified the following two main sulci in this region: the suprarostral sulcus (SU-ROS) and the superior rostral sulcus (ROS-S). An additional sulcus, ventral to the ROS-S, was observed in several subjects, and was labeled the inferior rostral sulcus (ROS-I).

Frontopolar sulci, identified as medial polar sulci (MPS) in the study by Mackey and Petrides (2014), were dissociated into the following three potential sulci: accessory supraorbital sulcus (ASOS), MPS, and supraorbital sulcus (SOS; see also Petrides, 2018). We distinguished them as follows: the MPS is formed by a fold in the frontopolar cortex creating a sulcus on the medial wall, while ASOS and SOS were defined as folds on the medial wall itself. SOS was more dorsal than ASOS and polar medial sulcus (PMS). An additional ventral medial polar sulcus (VMPS) was identified by the fact that it is the most ventral and directed toward the orbital part of the cortex, dorsal to the olfactory sulcus.

Although the cingulate cortex was not the focus of the present study, the cingulate sulci were also identified because they are necessary to define the morphological patterns of the ventromedial sulci. The main cingulate sulcus (CGS) was identified in all subjects, but the para-CGS (PCGS), a secondary sulcus, was sometimes observed either as a well marked sulcus or as a trace of it, which we labeled as spur-PCGS. Finally, we identify the subgenual intralimbic sulcus (SILS). This sulcus lies below the genu, hence the term "subgenual," and is located between the SU-ROS and the corpus callosum.

The cortical surface renderings were generated using the Connectome Workbench viewer (RRID:SCR_008750; http://www.humanconnectome. 
org/connectome/connectome-workbench.html; Marcus et al., 2011). To label each sulcus observed in the region of interest in every hemisphere, we examined the following three types of renderings of the medial wall: the volume $(\mathrm{T} 1 \mathrm{w})$; the mid-thickness surface; and the dilated surface with a projection of the curvature (Fig. 1). We then manually drew the sulcus on the dilated surface following the curvature projection.

Morphological analysis. All analyses and statistics were conducted in Matlab 2017a (MATLAB and Statistics Toolbox Release 2017a, MathWorks; RRID:SCR_001622; http://www.mathworks.com/products/ matlab/) with in-house bespoke scripts using Workbench executables. To compute the statistics and get information on each type of sulcus, regions of interest were created from the manual drawing explained above for every hemisphere. MNI coordinates (bic-mni-models, Montreal Neurological Institute; RRID:SCR_014087) were accessible in all of the surface files provided in the HCP dataset. For display purposes (see Figure $4 E$ ), we used a dilated version of the sulci ( $5 \mathrm{~mm}$ larger) for the analysis on the normalized length to increase the number of points entered in the analysis. The results did not change when the original sulcus was used as a region of interest (posterior vs anterior part weight comparison, $\left.t_{(113)}=-3.98, p=1.10^{-4}\right)$.

To compute the probability of observing one sulcus on one side given its presence on the other side, we used the following equation:

$$
P\left(S_{B} \mid S_{1}\right)=\frac{P\left(S_{1} \mid S_{B}\right) * P\left(S_{B}\right)}{P\left(S_{1}\right)}=\frac{P\left(S_{B}\right)}{P\left(S_{1}\right)}
$$

with $P\left(S_{B}\right)$ the probability of sulcus on both sides, $P\left(S_{1}\right)$ the probability of sulcus on only one side; and $P\left(S_{1} \mid S_{B}\right)=1$.

If not specified in the text, all comparisons were assessed by two-tailed $t$ tests. Analysis on volumes were conducted using the FMRIB (Functional MRI of the Brain) Software Library (FSL; RRID:SCR_002823).

Resting-state analysis. The rs-fMRI acquisitions (including the use of leading-edge, customized MRI hardware and acquisition software) and image processing are covered in detail in the studies by Glasser et al. (2013), Smith et al. (2013), and Uğurbil et al., 2013. After image preprocessing [primarily using the FSL (RRID:SCR_002823; Jenkinson et al., 2012); FreeSurfer (RRID:SCR_001847; Fischl, 2012), and Connectome Workbench (Marcus et al., 2013) software packages], the functional time series are filtered and artifacts are removed using an automated datadriven approach that relies on independent component analysis (ICA) decomposition and hand-trained hierarchical classification [FMRIB ICA-based X-noisifier (FIX); Smith et al., 2013].

After these image preprocessing and time series cleaning steps, the rs-fMRI data enter a probabilistic independent component analysis (MELODIC; Beckmann and Smith, 2004) to identify statistically independent spatial component maps and their accompanying time series. These spatial maps are not binary and can be overlapping: the value at each point in the brain can be considered a "weight" reflecting how strongly this point contributes to a given component. As a whole, each signal component map can be considered as a statistically independent probabilistic parcel reflecting a specific brain network. As such, the component weight maps are not the same as a seed-based correlation map, but are often interpreted in analogous ways.

To identify the component capturing the Default Mode Network, we examined them one by one for every subject and, for each session, we extracted the component that had the highest weights in the precuneus and posterior cingulate cortex (PCC), in bilateral posterior parietal cortex, and in bilateral temporal association cortex, following the definition provided by Raichle (2015). Weights correspond to the probability of each vertex belonging to a specific independent component. Note that to avoid any bias in our results, we ignored the weights on the medial wall of the prefrontal cortex (both the vmPFC and the dorsomedial prefrontal cortex). When several components were matching those criteria, we selected the one that was most bilateral. Components identified as DMN were finally averaged across sessions to obtain one DMN per subject.

Definition of the regions of interest. In the last section of the Results, we compare four ways of selecting a region of interest (ROI) in the vmPFC.

In the first method, called "a priori," the ROI was defined from a meta-analysis performed using Neurosynth (www.neurosynth.org;
RRID:SCR_006798) with the term "Default Mode Network" as a search term. The MNI coordinates of the peak of the Default Mode Network in the vmPFC were used to define the region of interest on each subject as a disc of diameter $10 \mathrm{~mm}$ around it. This ROI is an MNI-based ROI: vertices on MSMAll surfaces are different across subjects, but the MNI coordinates are the same.

The second and the third methods are labeled "circular inference" methods because they are selected on the basis of the group result and then tested (they will provide positive results by definition). The group ROI was defined according to the averaged DMN weight map across the group of subjects. We set an arbitrary threshold of weights to obtain a clear cluster in the vmPFC and then select this cluster as an ROI. This ROI is a vertex-based ROI (MNI coordinates are different across subjects).

The "peaks" ROI was based on the same map as the previous ROI but instead of selecting a cluster, the 10 vertices with the highest DMN weights were selected. MNI coordinates of those vertices were extracted and back-projected on each subject to extract the DMN weights at the subject level. This ROI is an MNI-based ROI.

Finally, the anatomical ROI, called the anterior ROS-S (aROS-S), was determined at the subject level based on the ROS-S drawn in the previous analysis. For each subject, we extracted the DMN weights within the anterior half of the ROS-S. This ROI is an anatomy-based ROI (MNI and vertices will be different across subjects).

For every subject, the DMN weights were extracted within each ROI and averaged across the ROI to get one value per ROI and per subject. Then, the significance of the differences between ROIs at the group level was assessed with paired $t$ tests.

To compare the quality of DMN identified with the "a priori" method and with the anatomical method, we defined three regions of interest representative of the DMN, as follows: the PCC, the anterior superior temporal sulcus (aSTS), and the temporoparietal junction (TPJ). We drew them manually on the map of averaged DMN weights (based on a threshold of 20 arbitrary units of weight).

Signal consistency corresponds to 1/STD_ROI, with STD_ROI corresponding to the $\mathrm{SD}$ of the time series across vertices within each ROI. A low SD indicates small differences of the signal across vertices of an ROI. We represented 1/STD_ROI in Figure 7.

\section{Results}

\section{Description of the sulci}

Using a subset of 57 subjects (i.e., 114 hemispheres) from the Human Connectome Project (see Materials and Methods), we identified all the possible sulci within the left and right hemispheres of the ventral part of the medial wall of the prefrontal cortex (i.e., the vmPFC). The following three categories of sulci were observed: the principal medial sulci; the supplementary medial sulci; and the polar/supraorbital sulci (Fig. 1, Table 1).

\section{Principal medial sulci}

The SU-ROS and the ROS-S were identified in all hemispheres. The mean average length of the SU-ROS was $36.87 \pm 0.73 \mathrm{~mm}$ with no significant difference between the left and right hemispheres (left, $36.71 \pm 0.94 \mathrm{~mm}$; right, $37.03 \pm 1.13 \mathrm{~mm}$; left vs right: $t_{(112)}=-0.21, p=0.83$, unpaired $t$ test). The ROS-S had an average length of $28.78 \pm 0.95 \mathrm{~mm}$ with a significant difference between the left and right hemispheres (ROS-S: left, $25.29 \pm 1.46$ $\mathrm{mm}$; right, $32.27 \pm 1.02 \mathrm{~mm}$; left vs right: $t_{(112)}=-3.92, p=$ $2.10^{-4}$, unpaired $t$ test). We did not find any significant correlation between the length of the sulci on the two hemispheres across subjects (SU-ROS: $r=-0.13, p=0.35$; ROS-S: $r=0.20$, $p=0.13)$

At the group level, we observed an overlap in the MNI locations of the center of gravity of those two sulci. The ROS-S average location was $y=48.20 \pm 0.61, z=-15.84 \pm 0.34$ on the left side, and $y=46.68 \pm 0.49, z=-14.84 \pm 0.29$ on the right side; the SU-ROS average location was $y=44.86 \pm 0.42, z=-8.78 \pm$ 
Table 1. Summary description of the vmPFC sulci

\begin{tabular}{|c|c|c|c|c|c|c|c|c|c|c|c|}
\hline Sulcus & ROS-I & ROS-S & SU-ROS & SILS & ASOS & SOS & VMPS & PMS & CGS & PCGS & $\overline{P C G S \text {-spur }}$ \\
\hline \multicolumn{12}{|l|}{ MNI coordinates } \\
\hline \multicolumn{12}{|l|}{ Barycenters } \\
\hline \multicolumn{12}{|l|}{ Left } \\
\hline \multirow[t]{2}{*}{$x$} & -8.53 & -7.15 & -6.57 & -4.26 & -9.20 & -9.26 & -9.73 & -10.55 & -6.24 & -7.02 & -7.46 \\
\hline & 0.12 & 0.12 & 0.12 & 0.10 & 0.13 & 0.12 & 0.16 & 0.19 & 0.11 & 0.15 & 0.20 \\
\hline \multirow[t]{2}{*}{$y$} & 54.60 & 48.20 & 44.86 & 39.09 & 61.81 & 62.35 & 58.34 & 65.61 & 33.71 & 46.52 & 53.13 \\
\hline & 0.40 & 0.61 & 0.42 & 0.45 & 0.28 & 0.30 & 0.37 & 0.28 & 0.61 & 0.94 & 0.71 \\
\hline \multirow[t]{2}{*}{$z$} & -17.18 & -15.84 & -8.78 & -3.19 & -6.96 & 5.02 & -16.42 & -0.51 & 27.89 & 27.15 & 22.26 \\
\hline & 0.28 & 0.34 & 0.46 & 0.82 & 0.56 & 0.67 & 0.43 & 0.51 & 0.50 & 1.06 & 1.16 \\
\hline \multicolumn{12}{|l|}{ Right } \\
\hline \multirow[t]{2}{*}{$x$} & 8.61 & 6.72 & 6.16 & 4.11 & 9.43 & 9.37 & 10.47 & 10.80 & 7.01 & 7.61 & 7.17 \\
\hline & 0.17 & 0.13 & 0.12 & 0.09 & 0.13 & 0.12 & 0.19 & 0.23 & 0.13 & 0.09 & 0.16 \\
\hline \multirow[t]{2}{*}{$y$} & 54.67 & 46.68 & 44.37 & 38.34 & 61.75 & 61.46 & 58.84 & 65.40 & 31.15 & 47.23 & 49.02 \\
\hline & 0.53 & 0.49 & 0.37 & 0.38 & 0.31 & 0.37 & 0.28 & 0.34 & 0.80 & 0.95 & 1.07 \\
\hline \multirow[t]{2}{*}{$z$} & -17.23 & -14.84 & -6.45 & -0.48 & -5.98 & 7.28 & -17.29 & 1.53 & 30.32 & 25.94 & 30.21 \\
\hline & 0.26 & 0.29 & 0.32 & 1.06 & 0.52 & 0.73 & 0.21 & 0.66 & 0.45 & 0.85 & 1.09 \\
\hline Top extremity & & & & & & & & & & & \\
\hline Left & & & & & & & & & & & \\
\hline$x$ & -9.48 & -8.33 & -8.50 & -4.98 & -10.18 & -9.78 & -9.62 & -11.34 & -5.94 & -5.71 & -6.21 \\
\hline & 0.14 & 0.14 & 0.13 & 0.15 & 0.16 & 0.15 & 0.21 & 0.24 & 0.15 & 0.11 & 0.27 \\
\hline$y$ & 59.16 & 57.55 & 56.51 & 44.73 & 64.75 & 64.78 & 58.97 & 67.06 & 7.51 & 30.12 & 42.96 \\
\hline & 0.27 & 0.47 & 0.41 & 0.75 & 0.26 & 0.36 & 0.40 & 0.27 & 1.35 & 1.87 & 2.01 \\
\hline$z$ & -14.85 & -11.39 & 0.21 & 4.74 & -4.91 & 7.66 & -15.37 & 1.29 & 47.55 & 45.78 & 35.89 \\
\hline & 0.38 & 0.60 & 0.67 & 0.99 & 0.61 & 0.69 & 0.46 & 0.53 & 0.70 & 1.28 & 2.05 \\
\hline Right & & & & & & & & & & & \\
\hline$x$ & 9.51 & 8.53 & 8.71 & 5.39 & 10.22 & 9.86 & 10.65 & 11.55 & 6.78 & 6.48 & 6.46 \\
\hline & 0.19 & 0.14 & 0.14 & 0.19 & 0.18 & 0.16 & 0.24 & 0.27 & 0.21 & 0.11 & 0.17 \\
\hline$y$ & 58.87 & 58.14 & 54.77 & 43.00 & 64.12 & 64.04 & 59.86 & 67.09 & 2.43 & 29.86 & 34.37 \\
\hline & 0.43 & 0.34 & 0.49 & 0.98 & 0.35 & 0.41 & 0.30 & 0.28 & 1.81 & 2.16 & 2.08 \\
\hline$z$ & -14.92 & -9.15 & 3.63 & 8.68 & -4.22 & 9.52 & -16.41 & 3.33 & 48.96 & 45.38 & 44.31 \\
\hline & 0.36 & 0.44 & 0.57 & 1.19 & 0.60 & 0.77 & 0.23 & 0.66 & 0.58 & 1.06 & 1.61 \\
\hline Bottom extrer & & & & & & & & & & & \\
\hline Left & & & & & & & & & & & \\
\hline$x$ & -7.80 & -6.02 & -4.78 & -3.56 & -8.41 & -8.79 & -9.22 & -9.49 & -6.61 & -8.24 & -8.43 \\
\hline & 0.14 & 0.15 & 0.12 & 0.10 & 0.12 & 0.11 & 0.18 & 0.16 & 0.19 & 0.18 & 0.16 \\
\hline$y$ & 49.54 & 38.07 & 32.06 & 30.09 & 58.12 & 58.77 & 56.81 & 63.05 & 49.44 & 55.18 & 58.84 \\
\hline & 0.68 & 1.06 & 0.66 & 0.69 & 0.42 & 0.40 & 0.41 & 0.44 & 0.53 & 0.58 & 0.35 \\
\hline$z$ & -19.43 & -19.05 & -14.96 & -8.71 & -8.96 & 2.42 & -17.07 & -2.12 & 4.64 & 4.52 & 5.81 \\
\hline & 0.24 & 0.25 & 0.40 & 1.20 & 0.56 & 0.69 & 0.41 & 0.52 & 0.72 & 1.29 & 0.62 \\
\hline Right & & & & & & & & & & & \\
\hline$x$ & 7.76 & 5.18 & 3.89 & 2.85 & 8.59 & 9.01 & 9.79 & 9.64 & 7.53 & 8.70 & 8.37 \\
\hline & 0.17 & 0.14 & 0.12 & 0.10 & 0.13 & 0.12 & 0.19 & 0.20 & 0.19 & 0.11 & 0.16 \\
\hline$y$ & 49.96 & 34.64 & 31.83 & 28.86 & 58.27 & 57.73 & 57.21 & 62.51 & 49.82 & 55.54 & 59.22 \\
\hline & 0.78 & 0.82 & 0.62 & 0.47 & 0.44 & 0.45 & 0.36 & 0.54 & 0.49 & 0.45 & 0.49 \\
\hline$z$ & -19.26 & -18.80 & -13.55 & -6.26 & -7.59 & 5.44 & -18.01 & 0.12 & 6.01 & 2.93 & 12.88 \\
\hline & 0.24 & 0.28 & 0.36 & 1.54 & 0.51 & 0.74 & 0.21 & 0.73 & 0.58 & 0.66 & 1.22 \\
\hline Characteristics (in & MNI space) & & & & & & & & & & \\
\hline Length & & & & & & & & & & & \\
\hline Left & & & & & & & & & & & \\
\hline Mean & 13.05 & 25.30 & 36.72 & 26.93 & 10.34 & 11.54 & 5.88 & 9.40 & 82.14 & 68.05 & 26.61 \\
\hline SEM & 0.78 & 1.46 & 0.94 & 1.08 & 0.57 & 0.58 & 0.32 & 0.57 & 2.73 & 2.28 & 3.60 \\
\hline Right & & & & & & & & & & & \\
\hline Mean & 12.54 & 32.28 & 37.03 & 30.78 & 10.61 & 11.36 & 5.69 & 8.78 & 88.32 & 66.41 & 43.09 \\
\hline SEM & 0.81 & 1.02 & 1.13 & 0.93 & 0.62 & 0.58 & 0.37 & 0.47 & 3.01 & 2.46 & 3.57 \\
\hline Depth & & & & & & & & & & & \\
\hline Left & & & & & & & & & & & \\
\hline Mean & -0.09 & -0.13 & -0.15 & -0.07 & -0.10 & -0.12 & -0.05 & -0.09 & -0.15 & -0.14 & -0.11 \\
\hline SEM & 0.008 & 0.008 & 0.004 & 0.008 & 0.007 & 0.006 & 0.011 & 0.011 & 0.003 & 0.003 & 0.005 \\
\hline Right & & & & & & & & & & & \\
\hline Mean & -0.10 & -0.16 & -0.14 & -0.10 & -0.11 & -0.13 & -0.06 & -0.10 & -0.14 & -0.13 & -0.09 \\
\hline SEM & 0.007 & 0.004 & 0.003 & 0.005 & 0.008 & 0.005 & 0.011 & 0.009 & 0.003 & 0.003 & 0.004 \\
\hline$\%$ of presence & & & & & & & & & & & \\
\hline Left & 52.63 & 100 & 100 & 64.91 & 91.23 & 100 & 31.58 & 91.23 & 100 & 61.40 & 10.53 \\
\hline Right & 75.44 & 100 & 100 & 22.81 & 96.49 & 100 & 47.37 & 94.74 & 100 & 54.39 & 22.81 \\
\hline All & 64.04 & 100 & 100 & 43.86 & 93.86 & 100 & 39.47 & 92.98 & 100 & 57.89 & 16.67 \\
\hline
\end{tabular}

Average (gray highlighted) and SEM (italic) MNI coordinates of the barycenter, top extremity, and bottom extremity of each vmPFC sulcus on each hemisphere across subjects. Length, depth, and percentage of presence are also reported. Top corresponds to the location of the most anterodorsal (posterodorsal for cingulate sulci) extremity of each sulcus. Bottom corresponds to the location of the most ventroposterior (anteroventral for cingulate sulci) extremity of each sulcus. PCGS-spur: spur paracingulate sulcus. 
0.46 on the left side, and $y=44.37 \pm 0.37, z=-6.45 \pm 0.32$ on the right side. However, on the dorsoventral axis, the range of location of the ROS-S on the left side varied from $z=-21$ to -9 $\mathrm{mm}$ and from $z=-16$ to $4 \mathrm{~mm}$ for the SU-ROS, indicating that, at the group level, there is an overlap in the MNI coordinates of these two sulci, notably between $z=-16$ and $-9 \mathrm{~mm}$. This result was also observed in the right hemisphere with a ROS-S range from $z=-20$ to $-7 \mathrm{~mm}$ and an SU-ROS range from $z=-12$ to $0 \mathrm{~mm}$.

\section{Supplementary medial sulci}

The ROS-I was present in $64.04 \%$ of the hemispheres, with $52.63 \%$ and $75.44 \%$ in the left and right hemispheres, respectively. The ROS-I was significantly more frequent in the right hemisphere compared with the left hemisphere $\left(t_{(112)}=-2.59\right.$, $p=0.01$, unpaired $t$ test). The ROS-I average location was $y=$ $54.60 \pm 0.40, z=-17.18 \pm 0.28$ on the left side, and $y=54.67 \pm$ $0.53, z=-17.23 \pm 0.26$ on the right side. The probability of having an ROS-I on both sides, given its presence on one side or the other, was estimated at $52.08 \%$, suggesting a relative independence of the two hemispheres regarding this sulcus.

The length of the ROS-I was on average $12.74 \pm 0.70 \mathrm{~mm}$ with no difference between the two hemispheres (left, $13.04 \pm 1.07$ $\mathrm{mm}$; right, $12.54 \pm 0.93 \mathrm{~mm}$; left vs right: $t_{(71)}=0.35, p=0.72$, unpaired $t$ test). There was no correlation of length between the two hemispheres when it was present on both sides $(r=0.11, p=$ $0.59)$.

The SILS was identified in $43.86 \%$ of the hemispheres, with $64.91 \%$ and $22.81 \%$ of the left and right hemispheres, respectively. The difference between hemispheres was significant $\left[t_{(112)}\right.$ $=4.96, p=3 \times 10^{-6}$, unpaired $t$ test; i.e., it was more probable to find this sulcus in only one hemisphere compared with the two hemispheres (the probability that SILS is on both sides given its presence on one side was 19.05\%)]. The average location of SILS was $y=39.09 \pm 0.45, z=-3.19 \pm 0.82$ on the left side, and $y=$ $38.34 \pm 0.38, z=-0.48 \pm 1.06$ on the right side. With an average length of $27.93 \pm 1.13 \mathrm{~mm}$, there was no significant difference of the SILS length between the two hemispheres $\left(t_{(48)}=-1.51, p=\right.$ 0.14 ) and no correlation of length for the cases in which SILS was found in both hemisphere $(r=0.27, p=0.52)$.

\section{Polar and supraorbital sulci}

The SOS was found in all hemispheres. The ASOS was present in $93.86 \%$ of the hemispheres with no over-representation in the right hemisphere: $96.49 \%$ in the right hemisphere and $91.23 \%$ in the left hemisphere $\left(t_{(112)}=-1.17, p=0.25\right.$, unpaired $t$ test $)$. Its existence on one side was predictive at $91.07 \%$ of its presence on both sides. The SOS average location was $y=62.35 \pm 0.30, z=$ $5.02 \pm 0.67$ on the left side, and $y=61.46 \pm 0.37, z=7.28 \pm 0.73$ on the right side. The ASOS average location was $y=61.81 \pm$ $0.28, z=-6.96 \pm 0.56$ on the left side, and $y=61.75 \pm 0.31, z=$ $-5.98 \pm 0.52$ on the right side.

The PMS, found in $92.98 \%$ of the hemispheres, was not more frequent in one hemisphere compared with the other (left, 91.23\%; right, 94.74\%; $t_{(112)}=-0.73, p=0.47$, unpaired $t$ test). Its existence on one side was predicted by its existence on the other side $(85.96 \%)$. The PMS average location was $y=65.61 \pm$ $0.28, z=-0.51 \pm 0.51$ on the left side and $y=65.40 \pm 0.34, z=$ $1.53 \pm 0.66$ on the right side.

Finally, we identified in some subjects a small sickle-shaped sulcus just dorsal to the olfactory sulcus in the anterior ventral polar region. This VMPS was observed in $39.47 \%$ of the hemi- spheres with a trend for a difference between the two hemispheres (left, $31.58 \%$; right, $49.12 \%$; left vs right: $t_{(112)}=-1.73, p=0.09$, unpaired $t$ test). The probability of having this sulcus in both hemispheres given its presence on one of the hemispheres was $36.36 \%$, suggesting that it is common to have this sulcus in only one hemisphere. The VMPS average center of gravity was $y=$ $58.34 \pm 0.37, z=-16.42 \pm 0.43$ on the left side, and $y=58.84 \pm$ $0.28, z=-17.29 \pm 0.21$ on the right side.

\section{Description of patterns}

We next examined the interactions between the different sulci. In agreement with Mackey and Petrides (2014), several interaction patterns between the SU-ROS and other sulci were identified. A fourth kind was also noted, but it represented $<5 \%$ of the subjects. We subdivided each group based on the presence or absence of a PCGS.

Type 1 corresponds to a merging of the SU-ROS with the CGS (subtype 1a) or with the PCGS (subtype 1b). In type 2, the SUROS merges with one or more polar sulci but not with the cingulate or paracingulate sulci. Subtypes were defined on the basis of the polar sulcus with which the SU-ROS merges (subtype 2a, SOS; subtype 2b, ASOS; subtype 2c, PMS; subtype 2d, merges with more than one polar sulcus). Type 3 refers to a merging of the SU-ROS with both the cingulate sulcus and a polar/supraorbital sulcus. Similar to subtypes identified in type 2, subtype 3 a merges with the cingulate sulcus while subtype $3 \mathrm{~b}$ merges with the paracingulate sulcus. These subtypes could also be separated in groups according to which polar sulcus the SU-ROS merges with (subtype 3a1: SOS; subtype 3a2, ASOS; subtype 3a3, PMS; subtype 3a4, two polar sulci; Fig. 2A). Type 4 corresponds to a merging with both cingulate and paracingulate sulci (subtype 4a). It could also merge with polar sulci (subtype 4b; Fig. 2B).

In agreement with Mackey and Petrides (2014), we found that $19.3 \%$ of all hemispheres belong to type $1,17.5 \%$ to type 2 , and $60.5 \%$ to type 3 . Type 4 was the smallest group with only $2.6 \%$ of hemispheres showing this pattern (Fig. 2C,D).

\section{Morphological deformation associated with sulcal pattern variability}

On the dorsoventral axis, we observed that the barycenters of the ROS-S and SU-ROS in the left hemisphere spread over 11 and 8 $\mathrm{mm}$, respectively (13 and $12 \mathrm{~mm}$ in the right hemisphere). To capture better how the interindividual variability in sulcal patterns impacts the organization of the vmPFC, we extracted for each subject the anteroposterior and dorsoventral MNI coordinates of the barycenter, the anterodorsal top extremity, the posteroventral bottom extremity, and the length of the ROS-S and the SU-ROS. We computed the partial correlation coefficients between the 14 variables ( $z$ and $y$ MNI for barycenter, top extremity, bottom extremity, and sulcal length for each sulcus) and the following six factors: absence or presence of the SILS, ROS-I, and PCGS; and the assignment to pattern 1,2, or 3. Significant influences were observed that were related to the presence of the SILS and ROS-I but not to the presence of the PCGS (all $p>0.05$ ). An effect of patterns 2 and 3 was also observed but nothing for pattern 1 (all $p>0.05$; Fig. $3 A$ ). We describe the significant results in the following sections.

\section{SILS}

The presence of the SILS had a strong influence of the MNI location of the SU-ROS because it induced a global mean shift of 3.1 $\mathrm{mm}$ (MNI space) of the SU-ROS toward the anteroventral direction. Indeed, the MNI $y$ and $z$ coordinates of the SU-ROS were 
A
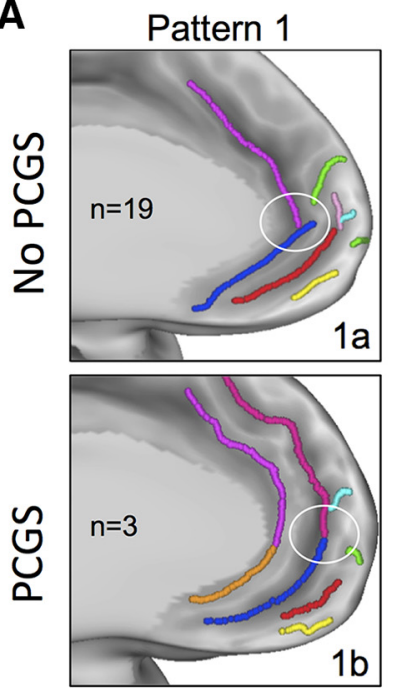

C

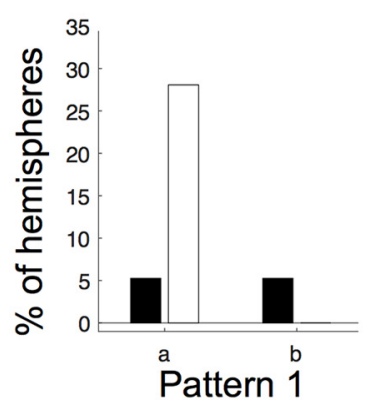

Pattern 2
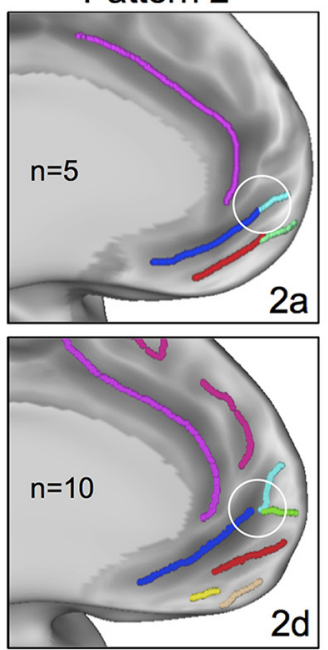

$2 \mathrm{~d}$

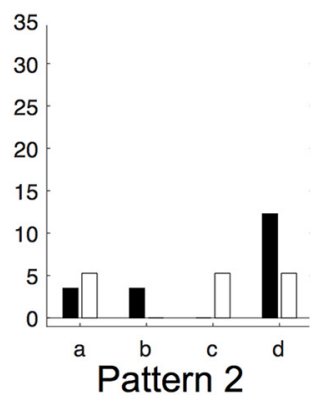

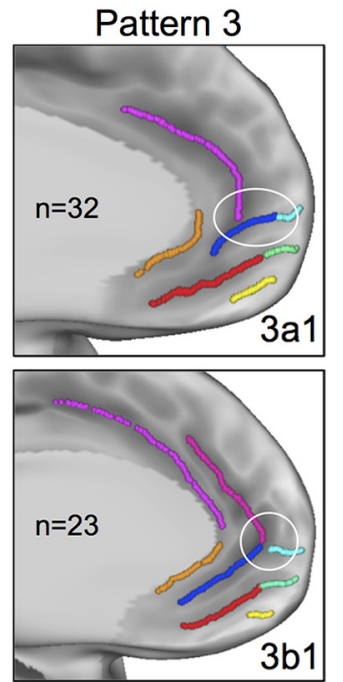

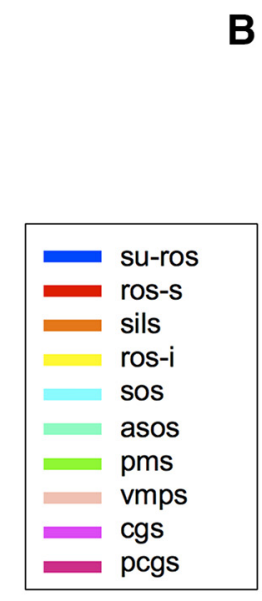

B
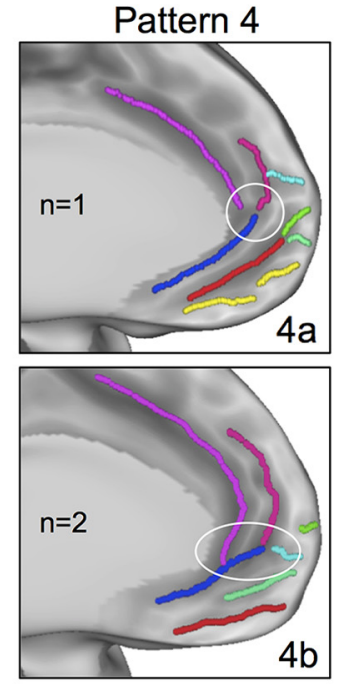

D
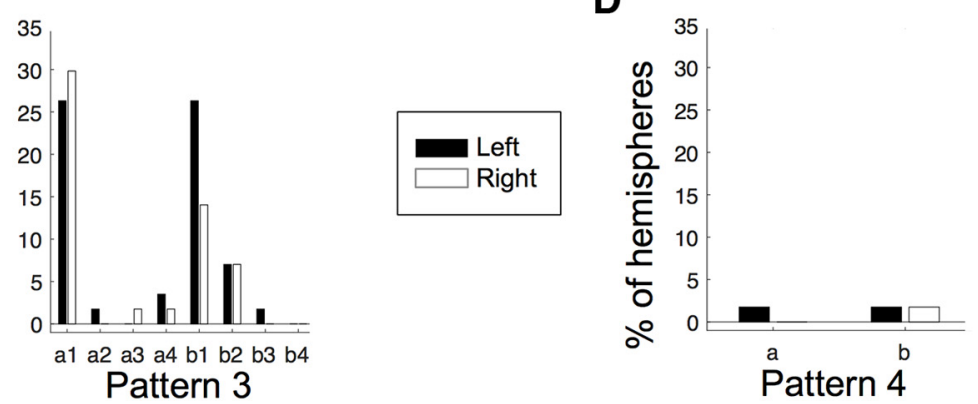

Figure 2. vmPFC anatomical patterns. $A$, Examples of the three common types of sulcal patterns in the vmPFC. The patterns depend on how the SU-ROS merges with other sulci (white circles) and the absence (first row) or presence (second row) of a PCGS. In type 1, the SU-ROS merges with either the CGS (1a) or the PCGS (1b). In type 2, the SU-ROS merges with one or more polar/supraorbital sulci but not with the CGS (2a) or the PCGS (2d). In type 3, the SU-ROS merges with both polar/supraorbital sulcus and CGS (3a1) or PCGS (3b1). B, In the rare type 4, the SU-ROS merges with both CGS and PCGS, without a merging (4a) or with (4b) a merging with a polar/supraorbital sulcus. $n$ indicates the number of hemispheres exhibiting each pattern. $C, D$, Percentage of left (black) and right (white) hemispheres displaying each pattern and subtype of patterns. 1a, only CGS (left, 5.3\%; right, 28.1\%); 1b, only PCGS (left, 5.3\%; right, 0\%); 2a, only SOS (left, 3.5\%; right, 5.3\%); 2b, only ASOS (left, 3.5\%; right, 0\%); 2c, only PMS (left, 0\%; right, 5.3\%); 2d, two polar/supraorbital sulci (left, 12.3\%; right, 5.3\%); 3a, with CGS and with SOS (3a1: left, 26.3\%; right, 29.8\%), or ASOS (3a2: left, 1.8\%; right, $0 \%$ ), or PMS (3a3: left, 0\%; right, 1.8\%), or with two polar/supraorbital sulci (3a4: left, 3.5\%; right, 1.8\%); 3b, with PCGS and with SOS (3b1: left, 26.3\%; right, 14.0\%), or ASOS (3b2: left, $7.0 \%$; right, $7.0 \%$ ), or PMS (3b3: left, 1.8\%; right, $0 \%$ ), or two polar/supraorbital sulci (3b4: left, 0\%; right, 0\%); 4a, CGS and PCGS (left, 1.8\%; right, $0 \%$ ); and 4b, CGS, PCGS, and polar/ supraorbital sulcus (left, $1.8 \%$; right, $1.8 \%$ ).

shifted for the barycenter ( $y$ : shift $=2.5 \mathrm{~mm}, \rho=0.35, p=2 \times$ $10^{-4} ; z$ : shift $\left.=1.8 \mathrm{~mm}, \rho=-0.21, p=0.03\right)$ and the top extremity $\left(y: \rho=0.28, p=3 \times 10^{-3} ; z: \rho=-0.29, p=2 \times\right.$ $\left.10^{-3}\right)$. The bottom extremity was also shifted toward the anterior direction $\left(y: \rho=0.30, p=1 \times 10^{-3}\right)$. Additionally, the ROS-S length was shorter when SILS was present $(\rho=-0.19, p=0.04$; Fig. 3B).

\section{ROS-I}

The presence of ROS-I had a strong impact on the MNI location of both principal sulci: the presence of ROS-I was dorsally shifting the barycenter of the ROS-S $(z$ : shift $=2.2 \mathrm{~mm}, \rho=0.39, p=$ $\left.3 \times 10^{-5}\right)$ and the SU-ROS $(z$ : shift $=2.7, \rho=0.34, p=3 \times$ $\left.10^{-4}\right)$. This dorsal shift could also be seen in a drift of the bottom and top extremities of both sulci (top: SU-ROS: $\rho=0.24, p=$ 0.01; ROS-S: $\rho=0.46, p=4 \times 10^{-7}$; bottom: SU-ROS: $\rho=0.30$, $p=2 \times 10^{-3}$; ROS-S: $\rho=0.21, p=0.03$ ). An additional effect of the presence of ROS-I was found on the ROS-S length: it was longer in hemispheres with ROS-I compared with hemispheres without it $\left(\rho=0.29, p=2 \times 10^{-3}\right)$. This was also observed in a significant effect on the anterior location of the ROS-S top extremity $(\rho=0.20, p=0.03$; Fig. $3 C)$.
As the presence or absence of ROS-I and SILS had opposite effects, we checked to what extent a hemisphere with ROS-I but without SILS would have a shift in its ROS-S and SU-ROS locations compared with a hemisphere without ROS-I but with SILS. The average anteroventral shift in MNI space was 3.5 and $4.2 \mathrm{~mm}$ for the ROS-S and the SU-ROS, respectively. The maximal dorsoventral distance observed between such two opposite hemispheres was $16.3 \mathrm{~mm}$ ( $9.3 \mathrm{~mm}$ for the anteroposterior direction). This result shows that the deformations associated with sulcal morphology can be critical for the interpretation of activity based solely on MNI coordinates compared with an anatomical referential.

\section{Patterns}

We did not observe a significant effect of the PCGS on the ROS-S or the SU-ROS morphology. We therefore merged the different subgroups of patterns based on the presence or absence of a PCGS to test for an effect of patterns on ROS-S and SU-ROS morphology. We only observed an effect on the SU-ROS anteroposterior location. Indeed, the SU-ROS in pattern 2 was more anterior (barycenter: shift $=2.4 \mathrm{~mm}, \rho=0.24, p=0.01$; top extremity: $\rho=0.23, p=0.02$ ). Pattern 3 also induced an anterior 
A

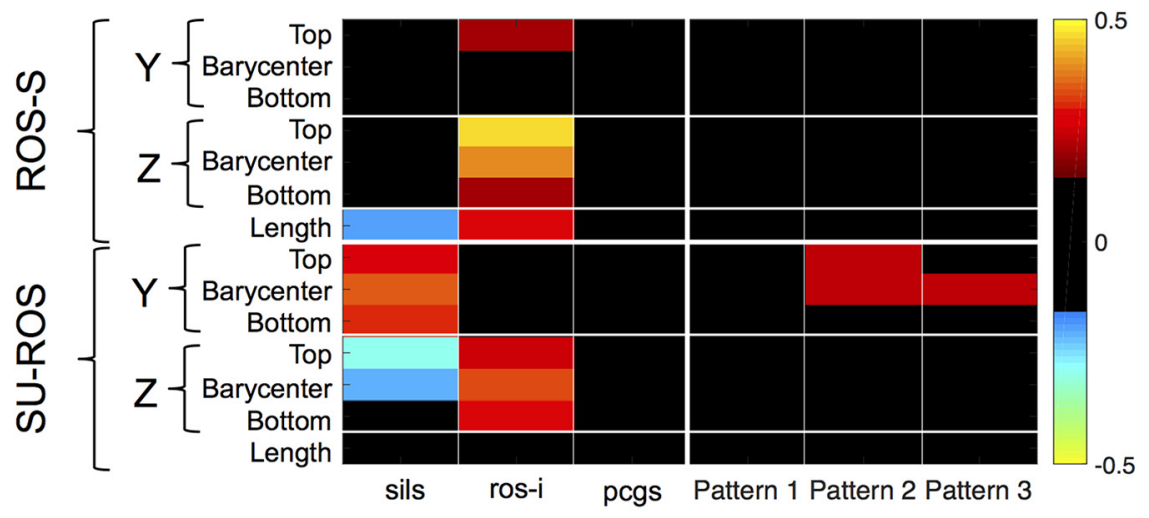

B
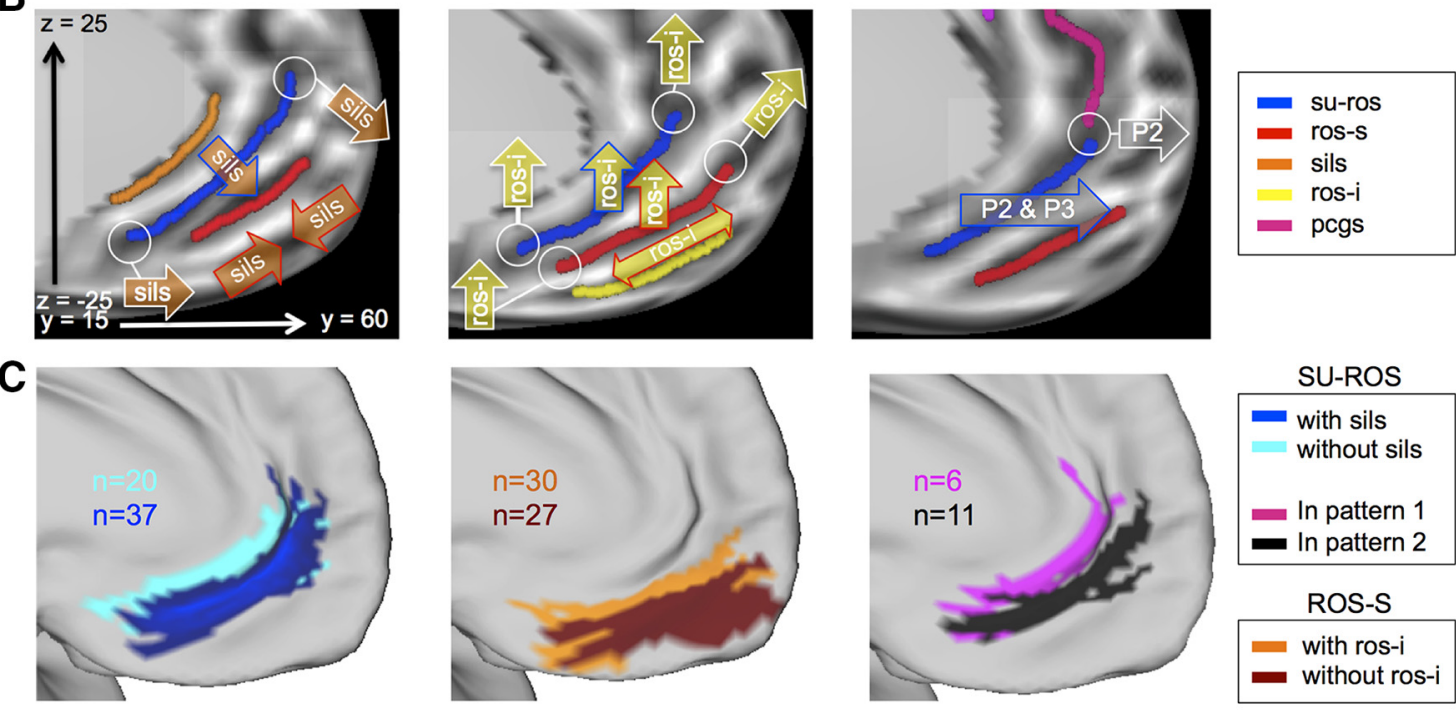

D

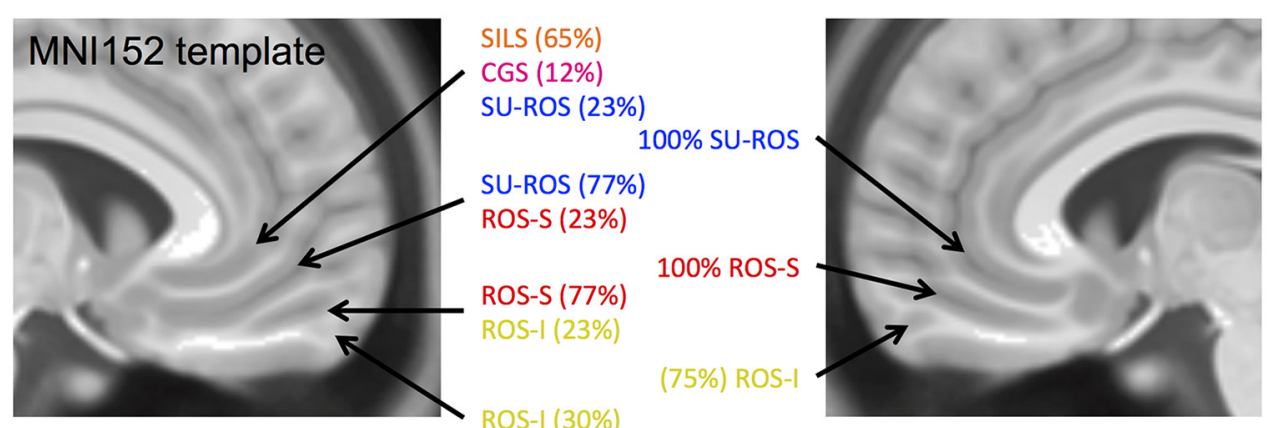

Figure 3. Morphological deformation associated with the presence of supplementary sulci and patterns. $A$, Partial correlation coefficients between each measure of interest of the ROS-S and SU-ROS, and the presence of SILS, ROS-I, PCGS, and patterns 1-3.y and z correspond to the anteroposterior and dorsoventral MNI coordinates, respectively. "Top" corresponds to the location of the most anterodorsal extremity of each sulcus. "Bottom" corresponds to the location of the most ventroposterior extremity of each sulcus. "Length" is the length of each sulcus. Only significant partial correlation coefficients are displayed $(p<0.05)$. Hot colors indicate a positive influence of having a supplementary sulcus or a specific pattern. Cold colors indicate negative effects. $\boldsymbol{B}$, Schematic representation of the significant influence of the presence of SILS (left), the presence of ROS-I (middle), and patterns 2 and 3 (P2 and P3, right) on the SU-ROS and ROS-S locations. Arrows attached to white circles indicate an influence on the circled extremity of the sulci; arrows with colored bordures indicating an influence on the barycenters of the sulci; double arrows indicate a positive effect on length while two facing arrows indicate a negative effect on length. C, Left, Projection of all the left SU-ROS for subjects with and without SILS. Middle, Projection of all the left ROS-S for subjects with and without ROS-I. Right, Projection of all the left SU-ROS for subjects with pattern 1 and subjects with pattern 2 . $n$ indicates the number of subjects displayed on the surface. $\boldsymbol{D}$, vmPFC sulci in the MNI152 template. On the left hemisphere, the sulci observed on the MNI152 template correspond to an overlap of several sulci. Percentages were computed from our sample of subjects. This overlap is induced by the morphological deformation caused by supplementary sulci. The right hemisphere of the MNI152 template does not present such overlaps because of the high presence of ROS-I. This absence of overlap does not mean that supplementary sulci do not induce morphological deformation on the right side; it indicates that the average template has sulci in the right hemisphere that globally correspond to the same right hemisphere sulci across subjects.

shift of the barycenter of the SU-ROS ( $\operatorname{shift}=2.0 \mathrm{~mm}, \rho=0.23$, $p=0.02$; Fig. 3D).

To characterize further the feature that had the greatest influence on the sulcal morphological variability in the vmPFC, we performed a principal component analysis on the 14 variables collected for every hemisphere. The first three components explained $84.1 \%$ of the variance in the data $(47.4 \%, 24.8 \%$, and $11.9 \%$ for the first, second and third components). We extracted those three first components and ran again the partial correlation analysis against the six features of interest. The results demon- 

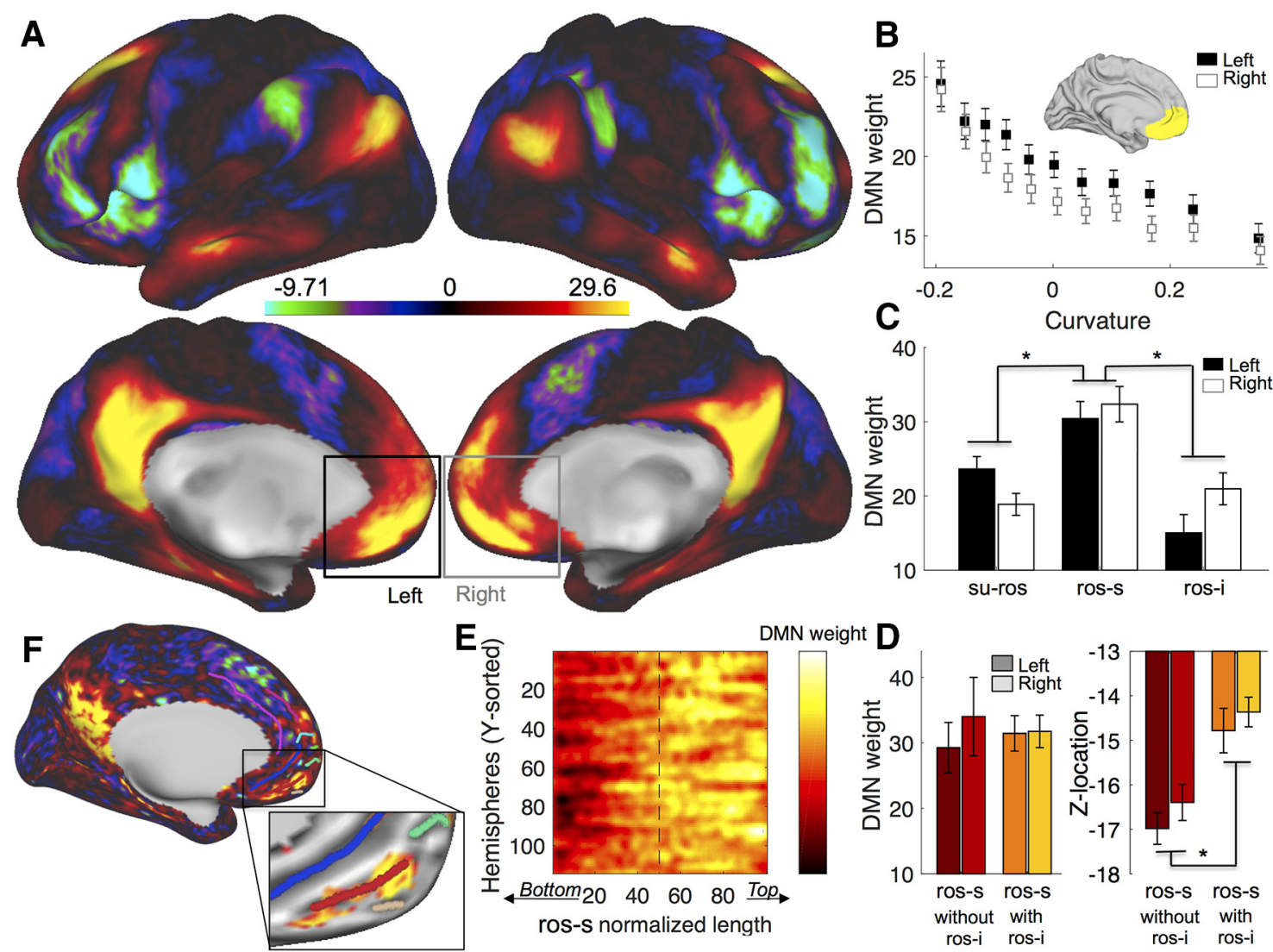

Figure 4. Default Mode Network weights and their dependence on the superior rostral sulcus. $\boldsymbol{A}$, Default Mode Network weights averaged across subjects on each hemisphere. $\boldsymbol{B}$, DMN weights are negatively correlated with the cortical curvature within the VmPFC (anatomical mask shown in the insert) for left (black) and right (white) hemispheres (squares represent binned data across hemispheres). Positive curvature corresponds to gyri, while negative curvature corresponds to sulci. C, Averaged DMN weights across subjects in the SU-ROS, the ROS-S, and the ROS-I in left (black) and right (white) sides. D, Averaged DMN weights (left) and z MNI location (right) in the ROS-S across subjects without ROS-I (red) and with ROS-I (orange) for left (dark color) and right (light color). Error bars represent the SEM. $\boldsymbol{E}$, Matrix of all ROS-S aligned on their normalized length and sorted according to their $y$ MNI location. Hot colors indicate high DMN weights. $\boldsymbol{F}$, Subject example of DMN with sulci. Insert is a zoom image of the $\mathrm{VmPFC}$ with an arbitrary threshold of the DMN weights. The strongest DMN weights are located on the anterior part of the ROS-S (red). Stars indicate significance ( $p<0.05$ ).

strated that the ROS-I was the only feature significantly affecting the first component $\left(\rho=0.31, p=9 \times 10^{-4}\right)$ and the SILS was the only feature affecting significantly the second component $\left(\rho=-0.27, p=5 \times 10^{-3}\right)$. The third component was significantly affected by the ROS-I presence $\left(\rho=0.29, p=2 \times 10^{-3}\right)$ but not by any other features (despite trends for pattern 2 and pattern 3, with $\rho=0.18, p=0.058$ and $\rho=0.17, p=0.077$, respectively). This result suggests that the ROS-I presence or absence can explain $>59.3 \%$ of the variability in the data compared with at least $24.8 \%$ for the SILS presence.

As a control analysis, we checked whether hemispheres with and without ROS-I had undergone different deformation during the normalization procedure of the brain volumes. We computed the deformation (curvature extracted from T1w undistorted images vs curvature extracted from T1w but resampled on MSMAll normalized) for each hemisphere and then compared it between hemispheres with and without ROS-I. No significant difference was observed, suggesting that the MSMAll normalization procedure did not affect differentially subjects with and without ROS-I. Consequently, morphological deformation induced by ROS-I presence cannot be attributed to higher artifactual deformation induced by volume normalization.

Finally, to illustrate the impact of our result, we investigated how the principal and supplementary sulci were represented in the MNI152 template (Fonov et al., 2009), conventionally adopted in FSL (Jenkinson et al., 2012) and SPM (Friston, 2007).
We registered original T1w volumes of our group of subjects on the MNI template (nonlinear registration), grouped subjects according to the presence or absence of ROS-I and SILS, and observed that the sulci observed on the right hemispheres corresponded to SU-ROS and ROS-S. However, on the left hemisphere of the template, the sulci do not correspond to a unique sulcus but to a mixture of sulci across individuals. Indeed, the first subgenual sulcus corresponds to an overlap of the SILS, the CGS, and the SU-ROS; the sulcus below this one corresponds to an overlap of the SU-ROS and the ROS-S; and the more ventral sulcus corresponds to an overlap between the ROS-S and the ROS-I (Fig. 3D). This phenomenon is primarily driven by the presence of ROS-I and SILS, which are close to $50 \%$ in the left hemisphere. Therefore, interpreting the location of activity in the left vmPFC with regard to the sulci of the MNI template might be misleading and careful conclusions should be drawn.

\section{Peak of default mode network related to sulcus location}

Finally, we tested whether the interindividual variability of the vmPFC sulcal pattern could have an impact on its functional organization. This analysis was based on rs-fMRI and more specifically on a network associated with the vmPFC, the DMN. The $\mathrm{DMN}$ is mainly composed of the vmPFC, the posterior cingulate cortex, and the lateral parietal cortex (Raichle, 2015). It can be easily identified during rs-fMRI by applying ICA. In the HCP dataset used in the present study, for each subject, the ICA com- 


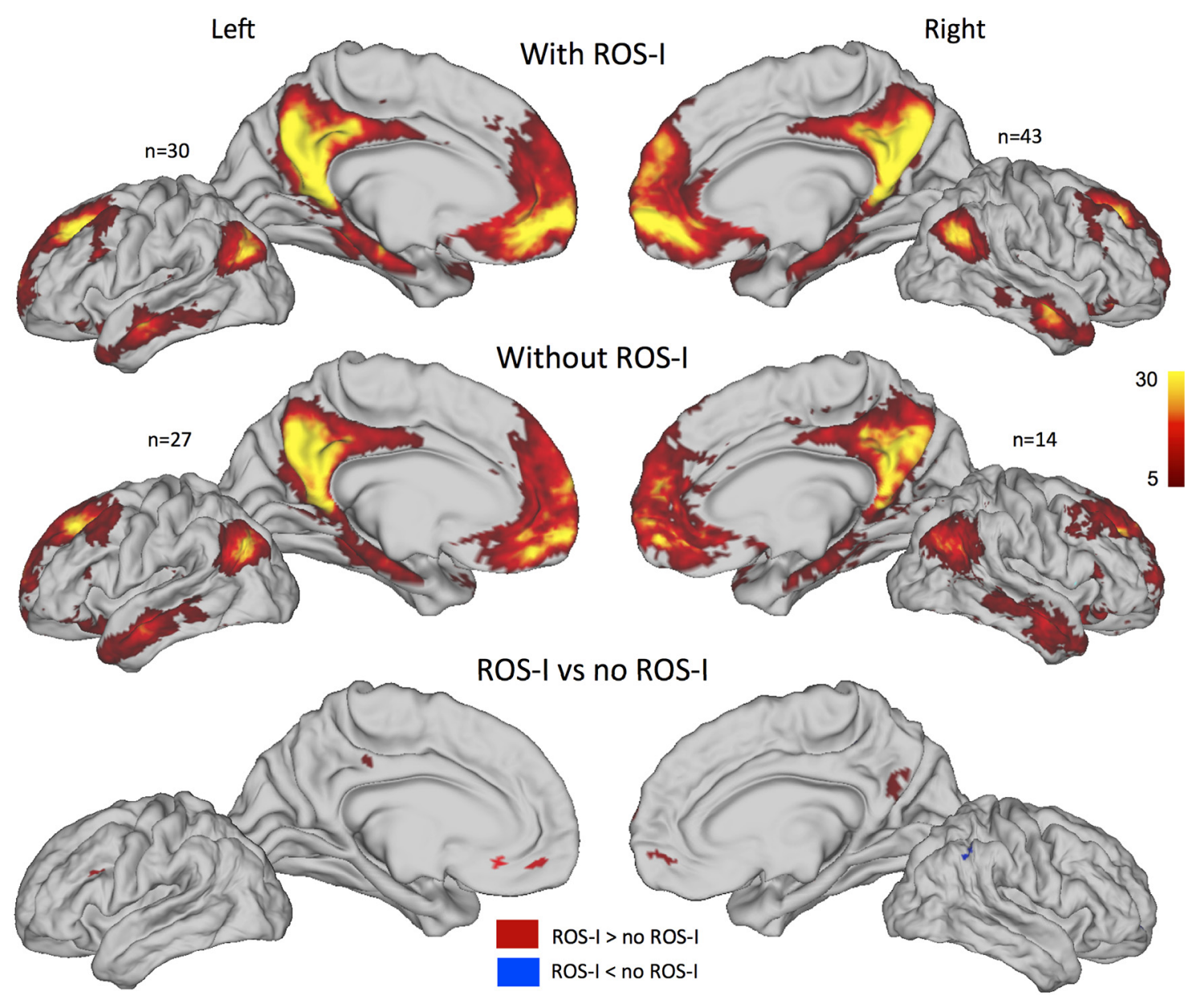

Figure 5. Default Mode Network weights in hemispheres with and without ROS-I. Default Mode Network (positive) weights averaged across subjects on each hemisphere for hemispheres with (top) and without (middle) ROS-I. $n$ indicates the number of hemispheres in each averaged map. The contrasts of the two groups for each hemisphere are displayed at bottom. Red (blue) clusters indicate higher (lower) weights for hemispheres with ROS-I compared with hemispheres without ROS-I. Clusters with a $t$ value $>2.7$ and $t$ values less than -2.7 and $>20$ mm ${ }^{2}$ are depicted.

ponent corresponding to the DMN was identified (see Materials and Methods). We then obtained for each subject a brain map of the DMN component. Each vertex of this map is associated with a weight, which corresponds to the probability of each vertex belonging to the DMN (Beckmann and Smith, 2004). An average of those weights is displayed in Figure $4 A$.

First, we determined whether vmPFC sulci-based ROIs would be relevant for the rs-fMRI analysis. We extracted the curvature (depth of the sulcus and gyrus) within a broad vmPFC mask (see methods). We regressed the DMN weights against the curvature within the vmPFC mask and found a strong negative effect across subjects (left: mean $=-19.1, \mathrm{SD}=2.12, t_{(56)}=-8.10, p=5 \times$ $10^{-11}$; right: mean $=-20.5, \mathrm{SD}=1.96, t_{(56)}=-8.54, p=1 \times$ $10^{-11}$; Fig. $4 B$ ), indicating that the DMN weights were stronger in deep areas, i.e., in sulci. Critically, this result indicates that the sulci within the vmPFC contribute more to the Default Mode Network than the gyri. In other words, a lack of correlation between a vmPFC sulcus-based ROI and DMN weights cannot be attributed to a poor sulcal MRI signal as opposed the one recorded on the gyrus.

We then extracted the DMN weights from the principal medial sulci (SU-ROS and ROS-S) and the ROS-I and tested for a difference between them. We found an effect of sulcus in a oneway $\operatorname{ANOVA}\left(F_{(2,297)}=18.7, p=2 \times 10^{-8}\right)$. Pairwise comparisons indicated that the ROS-S had significantly stronger weights than the SU-ROS $\left(t_{(226)}=5.1, p=8 \times 10^{-7}\right.$, unpaired $t$ test $)$ and than the ROS-I $\left(t_{(184)}=4.9, p=2 \times 10^{-6}\right.$, unpaired $t$ test $)$. No effect of side was observed (all $p>0.05$ ). Thus, at the group level, the ROS-S was identified as the main hub of the vmPFC for the Default Mode Network (Fig. 4C).

Given that the ROS-I had a strong influence on the location of the ROS-S, we checked the validity of the previous result with a complementary ANOVA including the following two factors: "group" and "sulcus." The group factor was defined as subjects with and without ROS-I (1/0), and the sulcus was defined as ROS-S and ROS-I (1/2). Again, we found a strong effect of sulcus $\left(F_{(1,183)}=20.11, p=1 \times 10^{-5}\right)$ but no effect of group $\left(F_{(1,183)}=\right.$ $0.04, p=0.85$; Fig. $4 D)$. This finding confirms the previous result showing that the ROS-S is a critical hub of the DMN independently of the existence of the secondary ROS-I sulcus.

Finally, we extracted the DMN weights within the ROS-S and sorted each vertex according to the $y$-axis. Given that each ROS-S across hemispheres had a different length, we normalized the length of the sorted vector to fit a 100 point vector. We then merged all the normalized vectors in a single matrix and observed that the strongest DMN weights were located in the anterior part of the ROS-S, starting at $\sim 50 \%$ of the ROS-S length (Fig. $4 E$ ). A simple comparison between the first half of the sulcus and the second half confirmed this observation $\left(t_{(113)}=-8.13, p=6 \times\right.$ $10^{-13}$, paired $t$ test). A representative hemisphere of this result is depicted in Figure 4F. Thus, we identified that the ROS-S, and its 


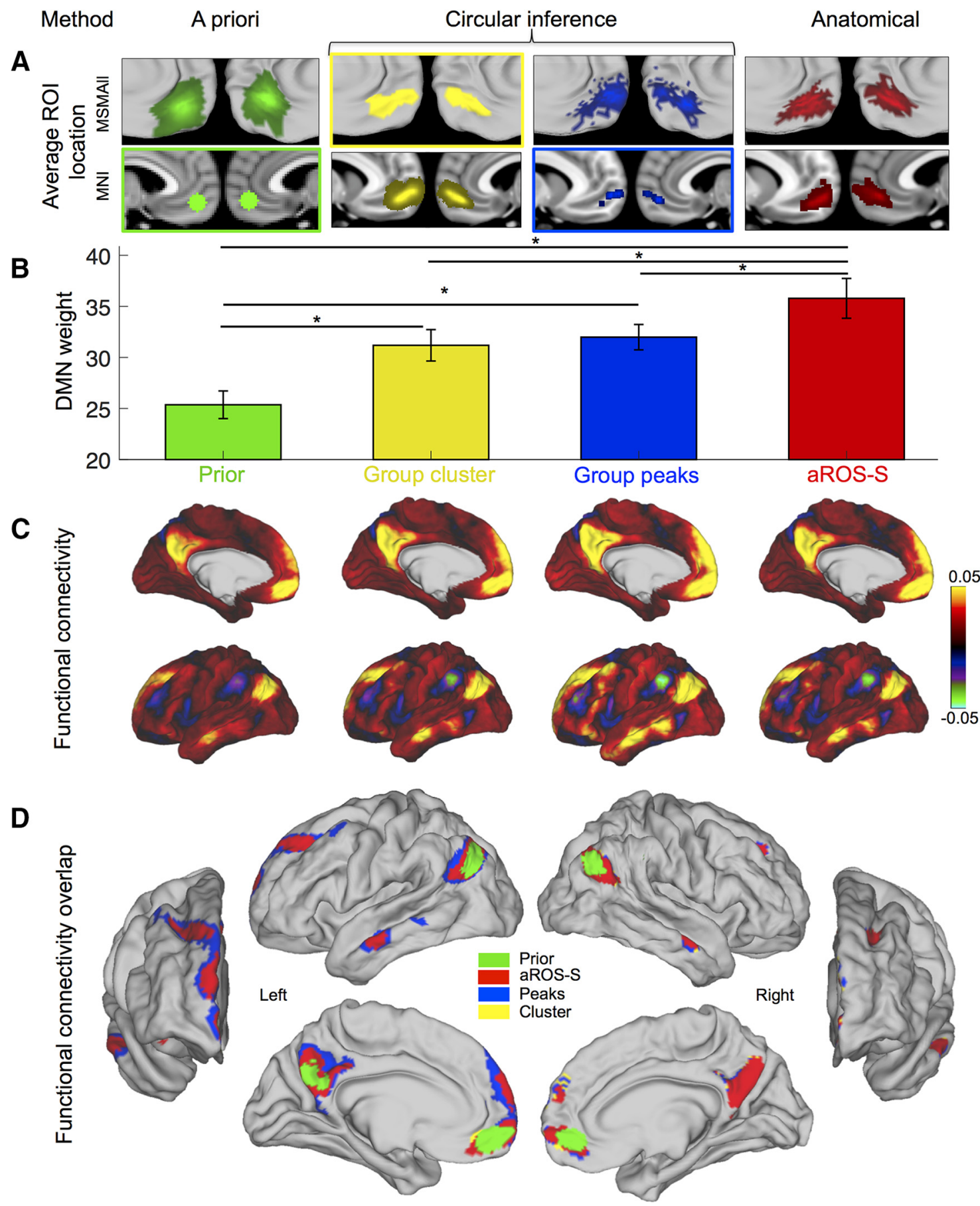

Figure 6. Default Mode Network weights in four vmPFC ROIs. DMN results from ROls defined according to four different methods: a priori MNI method (green, Prior), circular inference method, based on group results (cluster, yellow; peaks, blue), and anatomical method (aROS-S, red). A, Average ROl location across subjects on the MSMAll surfaces (top row) and in the MNI referential (bottom row). Colored rectangles indicate the referential of each ROI: MNI for Prior and Group peaks, vertices for Group cluster. There is no rectangle for the aROS-S because its referential is anatomical. B, Default Mode Network weights averaged across all hemispheres for each ROI. Error bars represent the SEM. Stars indicate significance. $\boldsymbol{C}$, Mean functional connectivity map across left hemispheres for each ROl. Colors indicate average correlation coefficients. Right hemispheres have a similar profile and are not displayed for illustrative purposes. $\boldsymbol{D}, 0$ verlap of the maps displayed in $\mathbf{C}$ but thresholded at 0.05 . Maps were superimposed according to the extent of the cluster (smaller above). The maps are displayed for left and right hemispheres with anterior, lateral, and medial perspectives.

anterior part in particular, is the hub of the vmPFC subcomponent of the DMN.

Given that ROS-I has a strong influence on the location of the ROS-S, one prediction following this result is that the DMN connectivity between subjects with and without ROS-I would be slightly different, especially in the vmPFC region. Indeed, if the vmPFC hub of the DMN is in the ROS-S, subjects with a ROS-I should have a stronger connectivity in the dorsal vmPFC. We averaged the DMN weights of subjects with and without ROS-I separately and then contrasted those maps (Fig. 5). We could observe that this prediction was confirmed in the vmPFC in which we found significant clusters $\left(>20 \mathrm{~mm}^{2}\right.$ and with $t$ values $>2.7$ ) in both left and right hemispheres. Moreover, a cluster also appeared in the PCC, suggesting that morphological variability in the vmPFC might also induce functional variability in distant regions such as the PCC. 


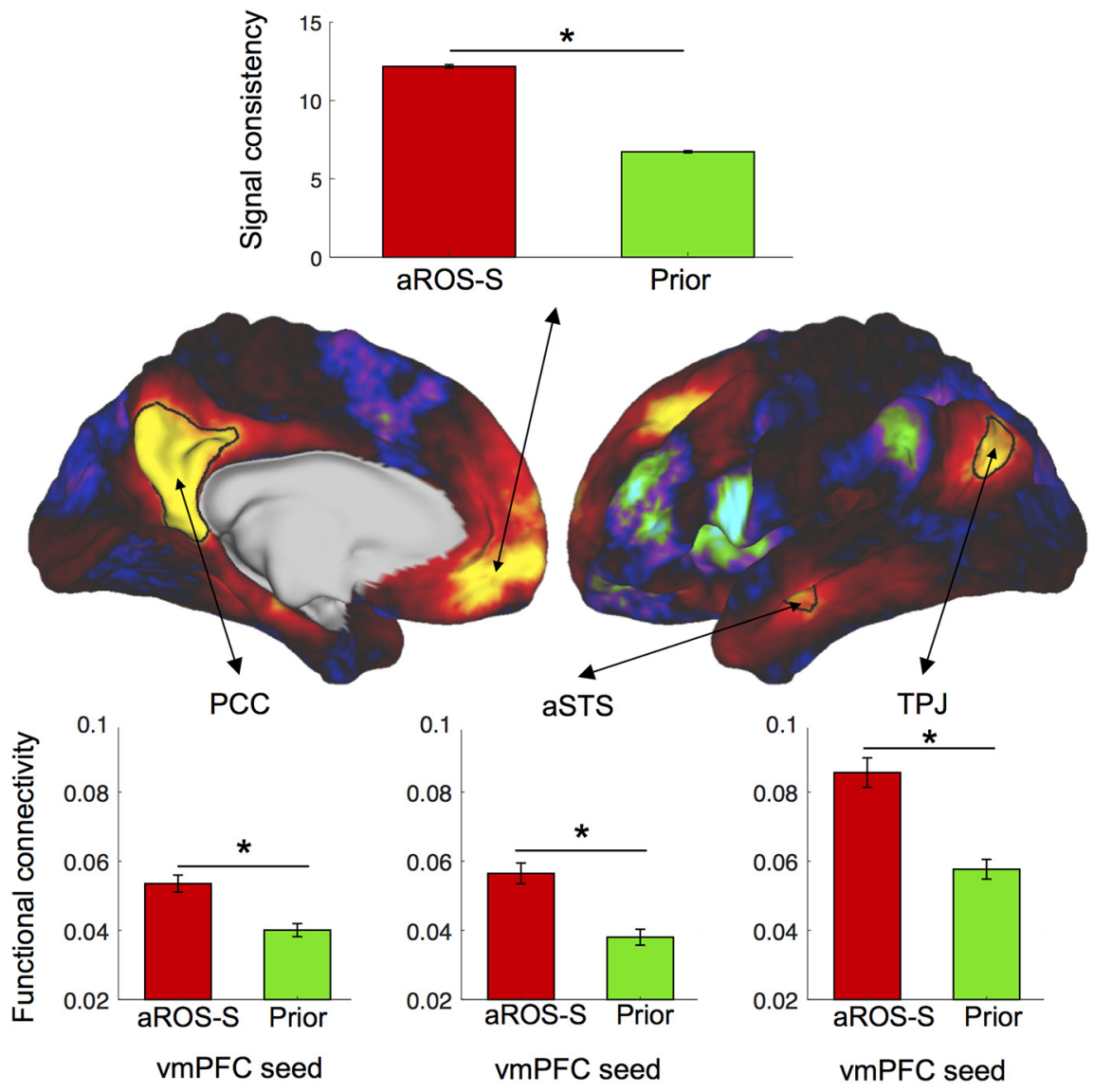

Figure 7. Default Mode Network components comparison between prior and anatomical ROI. Top, Signal consistency within aROS-S and Prior ROI. Signal consistency is the inverse of the SD of the raw signal across vertices averaged across time. Middle, Average Default Mode Network weights across left hemispheres (right hemisphere not shown for illustrative purposes). The map was used to draw the PCC, aSTS, and TPJ regions of interest. Bottom, Average functional connectivity across all hemispheres between the vmPFC seed (aROS-Sor Prior) and the PCC (left), the aSTS (middle), and the TPJ (right). Stars indicate significance $(p<0.05)$.

\section{Comparison of ROI definition methods to investigate the DMN}

As we intend here to show that taking into account morphological features such as sulcal location might help the investigation of specific functions of the vmPFC, we tested whether using a region of interest defined according to sulcal morphology was better than using a sphere around specific MNI coordinates. We compared four ways of selecting an ROI. The first way is an a priori ROI defined according to the peak of activity corresponding to the Default Mode Network term search in neurosynth.com ( $y=$ $50, z=-10)$ : we labeled it "Prior." The second and the third ways were defined from the average map of the Default Mode Network on the dataset used in our study and are depicted in Figure $4 \mathrm{~A}$. The second way corresponds to the cluster found on the average maps (Group cluster), and the third way corresponds to the 10 vertices with the highest DMN weights in the group (Group peaks). This procedure would be classically labeled as "double dipping" or "circular inference" since it is a selection of region following a positive result. It would bias the result in favor of a positive result. The fourth way is an anatomically based ROI: for each subject, we extracted the DMN weights of the aROS-S. A more detailed description of the ROI definition and weights extraction is included in the Materials and Methods. All four ROIs had significant DMN weights (all $p>0.05$ ). Not surprisingly, the Group ROIs showed a significantly stronger effect than the Prior ROI (Prior vs Cluster: $t_{(113)}=-6.43, p=3 \times 10^{-9}$; Prior vs
Peaks: $t_{(113)}=4.52, p=2 \times 10^{-6}$, paired $t$ tests). Interestingly we found that the anatomically defined ROI aROS-S had a significantly stronger effect than the three other ROIs (paired $t$ tests against the ROS-S ROI: Prior: $t_{(113)}=6.72, p=8 \times$ $10^{-10}$; Cluster: $t_{(113)}=2.74, p=7 \times$ $10^{-4}$; Peaks: $t_{(113)}=3.57, p=5 \times 10^{-4}$; Fig. 6). This result shows that using the anterior part of the ROS-S as a region of interest is more efficient at the group level than using MNI coordinates based on previous studies or on the group result. Thus, even when positively biasing an ROI selection, we have demonstrated that using anatomical information to select the ROI provides stronger results than using MNI coordinates as a reference.

Finally, we evaluated the extent to which selecting the aROS-S as a region of interest was advantageous. First, we used the four previously described vmPFC ROIs as seeds to compute the functional connectivity of each region with the rest of the brain. We observed a very similar pattern of connectivity for all of them. However, when those maps are thresholded at 0.05 and overlapped, we can observe differences. The DMN observed with the Prior ROI is the smallest, and the right PCC does not survive the thresholding. The Peaks and Cluster ROIs shared a very similar large network, while the DMN observed with the aROS-S ROI was more restricted even when covering the same brain region. Thus, the DMN elicited by the aROS-S ROI has the best specificity among the four ROIs, allowing a clear separation of functional areas with the strongest evidence.

Then, we quantified the advantage of using aROS-S as a vmPFC ROI compared with an a priori ROI for the investigation of functional networks (Fig. 7). We computed the average strength of connectivity of aROS-S and Prior with the main other components of the DMN (TPJ, PCC, and aSTS; see Materials and Methods for ROI definition) and compared them. We found that the functional connectivity of the aROS-S with the three components was stronger than the connectivity of the Prior ROI (PCC: $t_{(113)}=6.62, p=1 \times$ $10^{-9}$; TPJ: $t_{(113)}=7.76, p=4 \times 10^{-12}$; aSTS: $t_{(113)}=7.02, p=2 \times$ $10^{-10}$, paired $t$ tests). We then assessed the consistency of the recorded signal in the vmPFC ROI across the different vertices of each ROI. For each time point of the time series extracted from the aROS-S and the Prior, we computed the SD across all vertices forming each ROI and then averaged it across time and hemispheres. The signal consistency, corresponding to the inverse of this measure, was much stronger in the aROS-S (mean $=12.2 ; \mathrm{SD}=0.11)$ compared with the Prior ROI (mean $=6.7 ; \mathrm{SD}=0.07 ; t_{(113)}=-20.66, p=4 \times$ $10^{-40}$, paired $t$ test). Thus, by using the aROS-S as an anatomical ROI, we can have access to a more consistent signal in the vmPFC, but also to a well and clearly defined DMN.

\section{Discussion}

The present study examined the sulcal morphology of the vmPFC and its relation to functional organization as reflected in resting- 
state connectivity. We established that the ROS-S and the SUROS can be found in all hemispheres and can thus be considered as the two principal sulci of the vmPFC. Organized around these two principal sulci of the vmPFC, we identified the probability of the presence of the supplementary medial sulci, such as the ROS-I, the SILS, and the polar/supraorbital sulci (MPS, VMPS, SOS, ASOS). We then demonstrated that the presence or absence of three supplementary sulci, namely the SILS, the ROS-I, and PCGS, had a major impact on the morphology of the vmPFC. First, the presence of a SILS induces an anteroventral shift in the location of the SU-ROS. Second, the presence of ROS-I induces a dorsal shift of both the ROS-S and the SU-ROS. Third, the SUROS merges with the PCGS, or with the CGS when the PCGS is not present, and this pattern has an impact on the location of the SU-ROS, although the shifts associated with the presence of SILS and ROS-I were greater than those induced by the presence of the PCGS. Moreover, the transformations applied to the brain volume images for registration to the MNI152 template and to surface-based maps for MSMAll registration (functional and sulcal surface registration) can be affected by the interindividual variability in sulcal pattern. For example, we have shown that vmPFC sulci on the left hemisphere of brains registered to the MNI152 template do not correspond to the same sulci across subjects. This observation is of importance when considering interpreting the location of peaks of activity within the vmPFC.

The present results are consistent with those by Mackey and Petrides (2014), who examined cadaver brains in a smaller pool of subjects $(n=13)$. In our study, the suprarostral sulcus was observed in all hemispheres, while Mackey and Petrides (2014) identified it in $91.3 \%$ of the hemispheres. This difference can be explained by a quite rare yet observed merging between the superior rostral sulcus and the suprarostral sulcus. This merging can be observed either on the whole length of both sulci, resulting in a long and deep principal medial sulcus, or on a continuous merging between the anterodorsal extremity of the ROS-S and the ventroposterior extremity of the SU-ROS, resulting in an extremely long sulcus. Another noticeable difference is related to the SILS. We strictly used the SILS label only when no gyrus could be observed between the sulcus and the corpus callosum, and we report a higher proportion of the presence of SILS than Mackey and Petrides (2014) do (44\% against 30\%). MPS, SOS, and ASOS were observed in a high percentage of hemispheres, but we were able to identify an additional polar sulcus in $40 \%$ of the studied hemispheres. This sickle-shaped sulcus that occupies the most ventral part of the vmPFC is labeled as the VMPS. On average, its barycenter coordinates were $y=58, z=-16$. It is located just dorsal to the olfactory sulcus, and rostral to the ROS-I if an ROS-I is present.

Having determined the interindividual variability in sulcal patterns and its impact on the vmPFC morphology, we then assessed whether sulcal morphology could influence the functional organization of the vmPFC and whether it could be used to improve the characterization of functional activity observed in the vmPFC. For this purpose, we selected the Default Mode Network activation as a tool since the cluster of activity observed at the group level is covering a large part of the vmPFC. Importantly, we observed that the main hub of the Default Mode Network in the vmPFC is located in the anterior part of the ROS-S, regardless of the presence or absence of the secondary medial sulcus ROS-I. Note that the presence/absence of the ROS-I is variable across subjects and has a strong influence on the ROS-S location. In other words, the vmPFC peak of the functional DMN network is not optimally defined by stereotaxic MNI coordinates or by any other volumetric or surface-based group-averaged template. Instead, importantly, we have shown that the vmPFC peak of the Default Mode Network is best defined by an anatomical sulcal feature identified on an individual-by-individual basis.

The present results also raise methodological issues with regard to the investigation of the Default Mode Network, and to a greater extent of the function of the vmPFC. Indeed, as in the interpretation of the location of activity in the MNI space, using a region of interest based on MNI coordinates or on vertices can influence the results. Sulcal features might be more accurate and our results call for the development of tools allowing an automatic detection of such features to characterize better functional subdivisions of the vmPFC.

Previous studies had shown that the location of functional activity in fMRI can often be predicted by the local morphology of the sulci in the frontal cortex (Amiez et al., 2006, 2013; Amiez and Petrides, 2014, 2018; Li et al., 2015). Morphological features not only explain cross-subject variability in functional connectivity but also explain behavioral measures (Bijsterbosch et al., 2018). However, to the best of our knowledge, this is the first time that it is shown that the functional organization of the vmPFC in resting state can be predicted by its local sulcal morphology. It should be noted that Mackey and Petrides (2014) have examined the sulcal patterns of the vmPFC in relation to cytoarchitecture and demonstrated that there is a good relationship between the sulci and particular cytoarchitectonic areas. Based on the location of the DMN peak in the rostral part of the ROS-S, it is therefore likely that the core node of the DMN in the medial prefrontal cortex is associated with a granular cortex area $14 \mathrm{~m}$ or area $10 \mathrm{~m}$ (Bludau et al., 2014; Mackey and Petrides, 2014). Supporting this anatomical correspondence between the vmPFC DMN node and area $10 \mathrm{~m}$ is the fact that both have been functionally associated with supporting sociocognitive functions (Mars et al., 2012). Anterior cingulate cortex (ACC) contributions to the DMN have also been reported in the literature (Vincent et al., 2007; Mantini et al., 2011; Qin and Northoff, 2011). However, our results would suggest that the implication of the ACC to the DMN is related to the strong monosynaptic connections of cingulate 24 and 32 with the frontopolar cortex (Petrides and Pandya, 2007). Future investigation should now be directed to extending this finding to other task-related functional activity in other subdivisions of the vmPFC, such as in tasks examining responses to decision variables (Lopez-Persem et al., 2016) or subjective valuation (Lebreton et al., 2009).

In conclusion, the present study provides a description of the variability of the vmPFC sulcal morphology across individuals and demonstrates that this variability might reduce the anatomical precision of group average analyses. Thus, taking into account the local morphology of the vmPFC at the individual level to define regions of interest might be considered when assessing the multiple functions of this heterogeneous brain region.

\section{References}

Amiez C, Petrides M (2014) Neuroimaging evidence of the anatomofunctional organization of the human cingulate motor areas. Cereb Cortex 24:563-578.

Amiez C, Petrides M (2018) Functional rostro-caudal gradient in the human posterior lateral frontal cortex. Brain Struct Funct 223:1487-1499.

Amiez C, Kostopoulos P, Champod AS, Petrides M (2006) Local morphology predicts functional organization of the dorsal premotor region in the human brain. J Neurosci 26:2724-2731.

Amiez C, Neveu R, Warrot D, Petrides M, Knoblauch K, Procyk E (2013) The location of feedback-related activity in the midcingulate cortex is predicted by local morphology. J Neurosci 33:2217-2228. 
Bartra O, McGuire JT, Kable JW (2013) The valuation system: a coordinatebased meta-analysis of BOLD fMRI experiments examining neural correlates of subjective value. Neuroimage 76:412-427.

Beckmann CF, Smith SM (2004) Probabilistic independent component analysis for functional magnetic resonance imaging. IEEE Trans Med Imaging 23:137-152.

Bijsterbosch JD, Woolrich MW, Glasser MF, Robinson EC, Beckmann CF, Van Essen DC, Harrison SJ, Smith SM (2018) The relationship between spatial configuration and functional connectivity of brain regions. eLife 7:e32992.

Bludau S, Eickhoff SB, Mohlberg H, Caspers S, Laird AR, Fox PT, Schleicher A, Zilles K, Amunts K (2014) Cytoarchitecture, probability maps and functions of the human frontal pole. Neuroimage 93:260-275.

Bonnici HM, Chadwick MJ, Lutti A, Hassabis D, Weiskopf N, Maguire EA (2012) Detecting representations of recent and remote autobiographical memories in vmPFC and hippocampus. J Neurosci 32:16982-16991.

Chiavaras MM, Petrides M (2000) Orbitofrontal sulci of the human and macaque monkey brain. J Comp Neurol 422:35-54.

Evans AC, Collins DL, Mills SR, Brown ED, Kelly RL, Peters TM (1993) 3D statistical neuroanatomical models from 305 MRI volumes. In: 1993 IEEE Conference Record Nuclear Science Symposium and Medical Imaging Conference, Vol 3, pp 1813-1817. Piscataway, NJ: Institute of Electrical and Electronics Engineers.

Fischl B (2012) FreeSurfer. Neuroimage 62:774-781.

Fonov V, Evans A, McKinstry R, Almli C, Collins D (2009) Unbiased nonlinear average age-appropriate brain templates from birth to adulthood. Neuroimage 47:S102.

Friston KJ (2007) Statistical parametric mapping: the analysis of functional brain images, Ed 1. Amsterdam/Boston: Elsevier/Academic.

Glasser MF, Sotiropoulos SN, Wilson JA, Coalson TS, Fischl B, Andersson JL, Xu J, Jbabdi S, Webster M, Polimeni JR, Van Essen DC, Jenkinson M (2013) The minimal preprocessing pipelines for the human connectome project. Neuroimage 80:105-124.

Glasser MF, Coalson TS, Robinson EC, Hacker CD, Harwell J, Yacoub E, Ugurbil K, Andersson J, Beckmann CF, Jenkinson M, Smith SM, Van Essen DC (2016) A multi-modal parcellation of human cerebral cortex. Nature 536:171-178.

Hänsel A, von Känel R (2008) The ventro-medial prefrontal cortex: a major link between the autonomic nervous system, regulation of emotion, and stress reactivity? Biopsychosoc Med 2:21.

Jenkinson M, Beckmann CF, Behrens TE, Woolrich MW, Smith SM (2012) FSL. Neuroimage 62:782-790.

Lebreton M, Jorge S, Michel V, Thirion B, Pessiglione M (2009) An automatic valuation system in the human brain: evidence from functional neuroimaging. Neuron 64:431-439.

Li Y, Sescousse G, Amiez C, Dreher JC (2015) Local morphology predicts functional organization of experienced value signals in the human orbitofrontal cortex. J Neurosci 35:1648-1658.

Lopez-Persem A, Domenech P, Pessiglione M (2016) How prior preferences determine decision-making frames and biases in the human brain. eLife 5:e20317.

Mackey S, Petrides M (2014) Architecture and morphology of the human ventromedial prefrontal cortex. Eur J Neurosci 40:2777-2796.

Mantini D, Gerits A, Nelissen K, Durand JB, Joly O, Simone L, Sawamura H, Wardak C, Orban GA, Buckner RL, Vanduffel W (2011) Default mode of brain function in monkeys. J Neurosci 31:12954-12962.
Marcus DS, Harms MP, Snyder AZ, Jenkinson M, Wilson JA, Glasser MF, Barch DM, Archie KA, Burgess GC, Ramaratnam M, Hodge M, Horton W, Herrick R, Olsen T, McKay M, House M, Hileman M, Reid E, Harwell J, Coalson T, et al. (2013) Human connectome project informatics: quality control, database services, and data visualization. Neuroimage 80: 202-219.

Marcus DS, Harwell J, Olsen T, Hodge M, Glasser MF, Prior F, Jenkinson M, Laumann T, Curtiss SW, Van Essen DC (2011) Informatics and data mining tools and strategies for the human connectome project. Front Neuroinform 5:4

Mars RB, Neubert F-X, Noonan MP, Sallet J, Toni I, Rushworth MFS (2012) On the relationship between the "default mode network" and the "social brain.” Front Hum Neurosci 6:189.

Noonan MP, Walton ME, Behrens TE, Sallet J, Buckley MJ, Rushworth MF (2010) Separate value comparison and learning mechanisms in macaque medial and lateral orbitofrontal cortex. Proc Natl Acad Sci U S A 107: 20547-20552.

Passingham RE, Stephan KE, Kötter R (2002) The anatomical basis of functional localization in the cortex. Nat Rev Neurosci 3:606-616.

Petrides M (1996) Specialized systems for the processing of mnemonic information within the primate frontal cortex [and discussion]. Philos Trans Biol Sci 351:1455-1462.

Petrides M (2002) The mid-ventrolateral prefrontal cortex and active mnemonic retrieval. Neurobiol Learn Mem 78:528-538.

Petrides M (2018) Atlas of the morphology of the human cerebral cortex on the average MNI brain, Ed 1. New York: Academic.

Petrides M, Pandya DN (2007) Efferent association pathways from the rostral prefrontal cortex in the macaque monkey. J Neurosci 27:1157311586.

Qin P, Northoff G (2011) How is our self related to midline regions and the default-mode network? Neuroimage 57:1221-1233.

Raichle ME (2015) The Brain's default mode network. Annu Rev Neurosci 38:433-447.

Smith SM, Beckmann CF, Andersson J, Auerbach EJ, Bijsterbosch J, Douaud G, Duff E, Feinberg DA, Griffanti L, Harms MP, Kelly M, Laumann T, Miller KL, Moeller S, Petersen S, Power J, Salimi-Khorshidi G, Snyder AZ, Vu AT, Woolrich MW, et al. (2013) Resting-state fMRI in the human connectome project. Neuroimage 80:144-168.

Uğurbil K, Xu J, Auerbach EJ, Moeller S, Vu AT, Duarte-Carvajalino JM, Lenglet C, Wu X, Schmitter S, Van de Moortele PF, Strupp J, Sapiro G, De Martino F, Wang D, Harel N, Garwood M, Chen L, Feinberg DA, Smith SM, Miller KL, et al. (2013) Pushing spatial and temporal resolution for functional and diffusion MRI in the human connectome project. Neuroimage 80:80-104.

Van Essen DC, Ugurbil K, Auerbach E, Barch D, Behrens TE, Bucholz R, Chang A, Chen L, Corbetta M, Curtiss SW, Della Penna S, Feinberg D, Glasser MF, Harel N, Heath AC, Larson-Prior L, Marcus D, Michalareas G, Moeller S, Oostenveld R, et al. (2012) The human connectome project: a data acquisition perspective. Neuroimage 62:2222-2231.

Vincent JL, Patel GH, Fox MD, Snyder AZ, Baker JT, Van Essen DC, Zempel JM, Snyder LH, Corbetta M, Raichle ME (2007) Intrinsic functional architecture in the anaesthetized monkey brain. Nature 447:83-86.

Wallis JD (2011) Cross-species studies of orbitofrontal cortex and valuebased decision-making. Nat Neurosci 15:13-19. 